\title{
The DeDiMa battery: a tool for identifying students' mathematical learning profiles
}

\section{BACKGROUND}

The DeDiMa battery is designed for assessing students' mathematical learning profiles, and it has been used to validate a 4-dimensional model for classifying mathematical learning difficulties. The model arises from existing hypotheses in the cognitive psychology and neuroscience literature, while the DeDiMa battery provides a reliable set of mathematical tasks that help to match characteristics of students' mathematical performances to their more basic learning difficulties.

\section{PARTICIPANTS AND PROCEDURE}

In this report we address the question of how these tools can help sketch out a student's mathematical learning profile. The participants are $5^{\text {th }}$ and $6^{\text {th }}$ grade students.

\section{RESULTS}

We compare the emerging profiles of two students with mathematical learning difficulties (MLD) matched for age, performance on a standardized test, non-verbal IQ, and educational experiences. The profiles are very different.

\section{CONCLUSIONS}

We believe that this approach can inform the design of individualized remedial interventions for MLD students.

\section{KEY WORDS}

mathematical learning difficulties; mathematical learning; mathematical learning profile; dyscalculia

ORganizations - 1: Research Center for Psychophysiology and Education, University of Athens, Greece · 2: University of Modena and Reggio Emilia, Italy

AUthors' CONTRIBUtion - A: Study design - B: Data collection - C: Statistical analysis - D: Data interpretation .

E: Manuscript preparation - F: Literature search · G: Funds collection

CORRESPONDING AUTHOR - Giannis Karagiannakis, Ph.D., Research Center for Psychophysiology and Education, University of Athens, Deinokratous 27, 10675, Kolonaki, Athens, Greece, e-mail: G.Karagiannakis@primedu.uoa.gr

to CITE THIS ARTICLE - Karagiannakis, G., \& Baccaglini-Frank, A. (2014). The DeDiMa battery: a tool for identifying students' mathematical learning profiles. Health Psychology Report, 2(4), 291-297. DOI: 10.5114/hpr.2014.46329 


\section{BACKGROUND}

Mathematical competence implies a range of abilities associated with the sense of quantity, the decoding of symbols, memory, visual-spatial capacity, and reasoning. Students can experience difficulties in any of these areas or in their coordination. There are different communities of research currently active in investigating such difficulties, from cognitive psychology and neuroscience to mathematics education, but
Giannis Karagiannakis, Anna Baccaglini-Frank without having yet reached common grounds from which to develop further studies.

Most of the literature from the field of cognitive psychology investigates atypical development of basic number processing, and has introduced a number of terms and classifications that, as Geary and Hoard remark, seem in most cases to describe the same condition (2001). However, its definition is still a topic of debate (Fletcher, Lyon, Fuchs, \& Barnes, 2007; Mazzocco, 2008; Butterworth, 2010; Kaufmann et al., 2013), and, although a condition of "difficulties characterized by problems processing numerical information, learning arithmetic facts, and performing accurate or fluent calculations" is recognized in the DSM-V, there are still no consistent assessment tools (the cut-off scores vary from the $3^{\text {rd }}$ to the $32^{\text {nd }}$ percentile) applied across countries: in fact, prevalence varies from $1.30 \%$ to $13.80 \%$ (for example, Lanfranchi, Lucangeli, Jerman, \& Swanson, 2008; Watson \& Gable, 2013). This may be the case also because of the number of different hypotheses that have been advanced to explain underachievement in mathematics (Mazzocco, 2008; Kaufmann, 2008). Moreover, when taking into account the frequency of comorbidity and of heterogeneity, the situation becomes extremely complex (Kaufmann et al., 2013). Also very little is known on the actual implications of specific neurological deficits on the student's overall mathematical development. Over the last few years, an increasing body of research has proposed multi-deficit approaches as more appropriate for understanding developmental disorders or difficulties more in general, specifically including MLD (e.g. Fias, Menon, \& Szucs, 2013; Bartelet, Ansari, Vaessen, \& Blomert, 2014).

On the other hand, the field of mathematics education highlights how the construct of "learning disability" or "disorder" does not allow one to differentiate between difficulties that signal a stable disability in mathematics and those that are a result of deficient teaching experiences or lack of sufficient exposure (Heyd-Metzuyanim, 2012). In fact, although a variety of teaching materials, strategies and theories has been developed within the various communities of research involved, educators involved with "older" students (age 8 to 18) frequently find it hard to choose what to propose in each specific case.
Along these lines, we use the acronym MLD in place of "mathematical learning difficulties", as a broad category containing mathematics disabilities or severe difficulties. By no means do we intend to downplay the students' problems by proposing this terminology; instead our studies attempt to sort out important hypotheses from the fields of cognitive psychology and neuroscience and organize them with respect to possible educational practices in the teaching of mathematics, to help all students in need, as urgently pleaded for within the present panorama (Howard-Jones, 2011; Grabner \& Ansari, 2010).

To respond to this need, we developed a 4-dimensional classification model of MLD (Karagiannakis, Baccaglini-Frank, \& Papadatos, 2014), consisting of 4 domains: core number, visual-spatial, memory, reasoning; it can be used to outline the mathematical learning profile of a student, thanks to an appropriately designed computer-based assessment tool, the Detecting Difficulties in Mathematics (DeDiMa) battery, that contains a range of mathematical tasks. The DeDiMa battery should be complemented with additional psychometric tests (measuring the IQ, the executive system, the possible presence of ADHD, or of the autism spectrum...), to obtain a more complete cognitive mathematical profile of each student. This approach is in line with the DSM-V. The 4-dimensional model and the DeDiMa battery have proven to be valid and reliable (Karagiannakis, BaccagliniFrank, \& Roussos, under review). We hope these will be widely used internationally, leading to comparable data, on larger and larger samples, that can be used insightfully across countries and research communities.

The individualized mathematical learning profile (at a certain moment in time) reveals the student's weaknesses, but also his/her strengths, which can be used to design focused and personalized remedial interventions (see Karagiannakis \& Cooreman in press). We do not exclude that a student's profile may change over time; in future research we therefore propose to investigate possible students' evolutions by administering the DeDiMa battery at various phases of individualized teaching interventions.

The design and study of such teaching interventions is a main direction of our ongoing research. In particular, we are exploring the potential of the constructed framework for adapting existing and designing new didactical material and activities, matching students' profiles. We are pursuing these directions with the explicit hope of crossing the boundaries of different fields of research, of informing all interested communities of research and fostering dialogues, and eventually of creating common scientific grounds.

In the following section we briefly describe the DeDiMa battery and other psychometric measures that we have used to identify the mathematical learning profiles of more than 300 students so far. Then 
we will answer the main question, the focus of this paper, by giving specific examples of students' mathematical learning profiles:

How can a student's mathematical learning profile be identified through the DeDiMa battery (complemented with other cognitive measures) and the 4-dimensional model?

\section{THE DeDiMa BATTERY}

The battery of tasks ${ }^{1}$ is computer-based and it consists of 13 tasks, for $5^{\text {th }}$ and $6^{\text {th }}$ grade students (age 10-12). Although there is no time limit, none of the over 300 students to whom the battery has been administered took more than 40 minutes to complete it. We briefly describe the tasks below ${ }^{2}$.

1. Subitizing-Enumeration (56 stimuli): The student is asked to compare a random array of dots shown on half of the computer screen to an Arabic digit shown on the other half of the screen. The stimuli are to be completed as quickly as possible.

2. Number magnitude comparison (64 stimuli): Two numbers from 1 to 98 in Arabic digits are simultaneously displayed on the computer screen. The student is asked to select the larger number as quickly as possible.

3. Dots magnitude comparison (28 stimuli): The student is presented simultaneously with two arrays of dots and asked to select the one that contains more dots (see Gebuis \& Reynvoet, 2011).

4. Addition fact retrieval (33 stimuli): The student is simultaneously presented with a single-digit addition (with operands between 2 and 9) that appears in the center of the screen, with two possible answers underneath (of which only one is correct), and is asked to choose the right answer as quickly as possible.

5. Multiplication fact retrieval (34 stimuli): The student is simultaneously presented with a single-digit addition (with operands between 2 and 9) that appears in the center of the screen, with two possible answers underneath (of which only one is correct), and is asked to choose the correct answer as quickly as possible.

6 and 7. Number Lines 0-100 - Ordinality (11 stimuli): A series of 22 number lines, in pairs, containing a blank line with two endpoints (0 and 100) are presented to the student on the computer screen, together with a target number (e.g., 29) above the center of each line. In this Number to Position task (see Siegler \& Opfer, 2003) the student is asked to consider the first number line (the one on top) and click on the position where the target number (above it) should lie (for a detailed description, see Siegler \& Booth, 2004). Then, the student is asked to perform the same task on the second number line (below it and aligned with it), placing the second target number on it. As this task is carried out, the first estimated position remains on the screen.

8. Number Lines $0-1000$ (11 stimuli): This task is analogous to Number Lines $0-100$, except that each line is presented alone and it is from 0 to 1000 .

9. Math Terms (30 stimuli): The student is presented with a shape or a number in the center of the screen, in red, and with three math terms underneath, and is asked to choose the term which corresponds to the red stimulus by clicking with the mouse on one of the possibilities.

10. Calculation principles (10 stimuli): The student is instructed to type a number into a gap in an equation that appears horizontally in the center of the computer screen, above a second completed equation. The number to be typed into the first equation can be obtained without computation, using the "relevant principle" introduced in the second equation.

11. Mental calculation (10 stimuli): The student is instructed to type a number into the gap of an equation that appears horizontally in the center of the computer screen. Unlike for the Calculation principles task, in this case no other equations appear on the screen.

12. Equations (10 stimuli): The student is asked to fill the gap in an equation containing numbers with 1 to 3 digits. To fill the gap the student has to click on the gap and select from a menu of possible answers. Only one answer is correct for each equation, and it can be a number or the math symbols of an operation.

13. Word problems (13 stimuli): The student is asked to solve everyday life story problems involving addition, subtraction, multiplication, and division. Once the DeDiMa battery has been completed on the computer, output is given in the form of a bar chart in which the student's Stanine Score (Thorndike, 1982) for each task is shown. Scores from these tasks are taken into consideration for the student's qualitative profile, together with additional cognitive measures. Principal component analysis (varimax) revealed that tasks $8,9,10,11,12,13$ assess the student's reasoning domain, tasks 4 and 5 the memory (retrieval) domain, tasks 1, 2, 3 the core number domain, and tasks 6 and 7 the visual-spatial domain. Confirmatory Factor Analysis also ratified the aforementioned components (Karagiannakis et al., under review). In Figure 1 the tasks are grouped according to these components, in the order of importance of the components revealed by the analyses.

Additional cognitive tests for complementing the student's profile: We additionally proposed a visual matrix span task (additional task A), a written calculations task (additional task B), and a reading fluency standardized test (Test A, by Panteliadou \& Antoniou, 2008) (additional task C).
The DeDiMa battery 


\section{IDENTIFYING STUDENTS' MATHEMATICAL LEARNING PROFILES}

In this section we explain how a student's mathematical learning profile can be identified through the DeDiMa battery (complemented with the other psychometric measures described) and the 4-dimensional model (Karagiannakis et al., 2014) presented in the introduction. The profiles provide a tool for gaining insight into a student's strengths and weaknesses in

Giannis Karagiannakis,

Anna Baccaglini-Frank learning mathematics, and they are much more personalized than a score on a standardized test. To show this, we now provide a comparison example of two very different mathematical learning profiles (based on performance on the DeDiMa battery) belonging to students of the same age, with matching performance on the NUCALC standardized arithmetic test (Koumoula et al., 2004) and matching non-verbal IQ. The profiles are part of the data collection from a group of 165 students in the $5^{\text {th }}$ and $6^{\text {th }}$ grade, recruited from 4 Greek schools, and whose supervisors signed informed consent forms for the study. Moreover, the profiles compared are of two students, a male and a female, who had been in the same classroom through elementary school, so we can suppose their mathematical exposure to be similar.

\section{A COMPARISON OF TWO SETS OF RESULTS FROM THE DEDIMA BATTERY}

Below is a graph comparing the students' Stanine Scores (from 0 to 9 , where 5 is the mean, and 2 is standard deviation) on the tasks of the DeDiMa battery and on the additional cognitive tests (Figure 1).

The scores clearly show that the students have different performances with respect to the different domains of the model. Their only similarity is the low performance with respect to the domain of reasoning. In the following section of the paper we will advance a hypothesis as to why this may be the case. In particular, the students' performances show that with respect to the domain of:

- reasoning: both students have a low performance score on these tasks, with the difference that student 1's results are particularly low on task 12 , but somewhat better on task 13. On tasks 8,9 and 10 the performance of student 1 is very low, while stu-

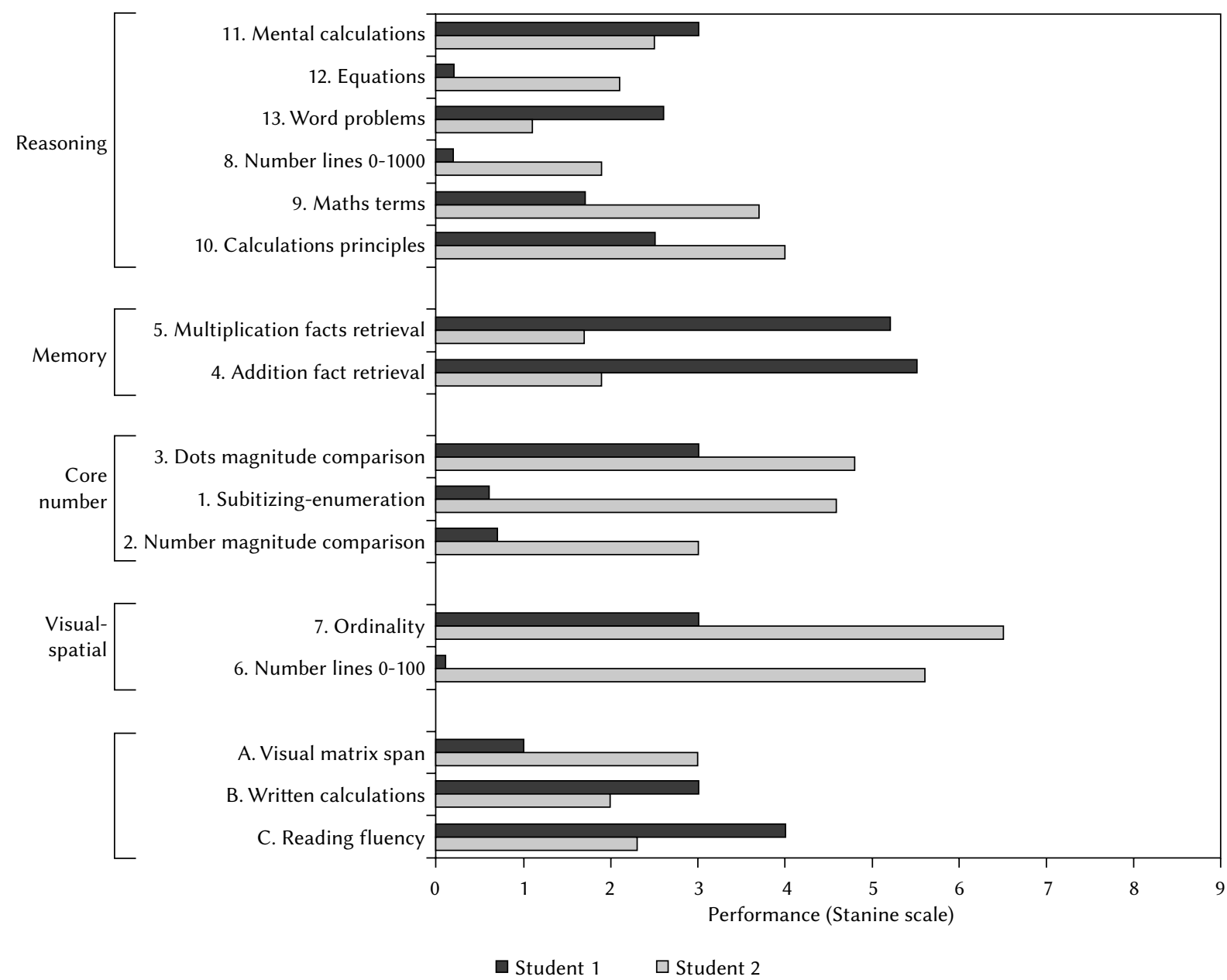

Figure 1. The comparison of Stanine Scores of two students on the DeDiMa battery and on the other psychometric measures. 
dent 2's performance is better, and typical on tasks 9 and 10 ;

- memory (fact retrieval): student 1 has an average performance, while student 2 performs very poorly on these tasks (4-5 of the DeDiMa battery);

- core number: student 1 has very low scores on tasks 1 and 2; student 2, instead, shows normal performance on tasks 1 and 3, with a slightly lower performance on task 2;

- visual-spatial: student 1 has an overall very low performance, while student 2's performance is within normality.

Analogously, student 2's performance is higher than student 1's on the visual matrix span task, while on written calculations the performance of student 1 is better than that of student 2 , and on reading fluency student 1 has a typical performance, while student 2 has a very low performance.

\section{INTERPRETATION OF THE RESULTS TO OUTLINE THE STUDENTS' MATHEMATICAL LEARNING PROFILES}

These results allow us to form initial hypotheses on the students' mathematical learning profiles.

a) Student 1's profile presents difficulties with Arabic symbols and making sense of numbers (see his performance on tasks 1 and 2), possibly due to a core number deficit and/or limited visual-spatial working memory (see performance on the additional task A). On the other hand, he shows strong fact retrieval skills (see his performance on tasks 4 and 5), possibly due to sufficient long-term memory.

b) Student 2 has difficulty in retrieving (perhaps also in saving to long-term memory) numerical facts (see her performance on tasks 4 and 5). On the other hand, she presents efficient number sense and visual-spatial abilities (see her performance on tasks 1, 2, 3, 6, 7).

These initial hypotheses can be used as a guide for analyzing other aspects of the students' performance. The analyses may lead to confirmation or to re-elaboration of the hypotheses.

Mental calculation: Both students perform poorly on this task, but the reasons may be different. The performance of student 1 can be explained by his core number deficit, which leads to difficulties in recognition and elaboration of the meaning of digits, as well as by difficulties with basic arithmetical principles (task 10 of the DeDiMa battery) and, possibly, by limitations in his visual-spatial working memory (additional task A). Although the student's reasoning skills seem to be lower than those of student 2 , the fact that his performance is slightly better on this task may be due to his efficient fact retrieval skills. On the other hand, the performance of student 2 can be explained by her deficit in numerical fact retrieval. We expect that a more qualitative clinical interview would have revealed the use of appropriate strategies, but of incorrect use of number facts within such strategies. This hypothesis seems to be confirmed by the student's normal performance on the calculations principles task (task 10 of the DeDiMa battery).

Equations: In this task, managing Arabic digits and operation signs is involved, so student 1 probably has a poor performance due to his inability to make sense of the digits and operation signs he sees. This hypothesis is also supported by the student's very low performance on tasks 3 (number magnitude comparison), 6 and 7 (visual-spatial domain) of the DeDiMa battery. Student 2 performs somewhat better on this task, because she can probably make sense of the equations, but her performance is still well below average, possibly because of the incidence of numerical fact retrieval on this task.

Word problems: In this task, student 1 has a slightly better performance than student 2, possibly because his typical reading skills allow him to feel comfortable with the texts. However, his overall performance is below average, possibly because of his core number deficit and difficulties with the meaning of mathematics vocabulary (task 9 of the DeDiMa battery). Student 2 has an extremely low performance, probably due to her low reading skills and difficulty in combining the numbers in the text according to the correct number of facts to reach the final answers. She may also have low phonological working memory, which often is associated with reading difficulties.

Written calculations: Both students perform poorly, but probably, again, for different reasons. Student 1 , as we suggested, may be unable to make sense of the numbers he sees and to re-elaborate them meaningfully, checking to see whether the final answer makes sense. In fact, the student seems to have great difficulties with estimation of larger numbers (task 8 of the DeDiMa battery). Moreover, his visual-spatial difficulties (tasks 6 and 7 of the DeDiMa battery) may account for problems with the visual-spatial components present in this task (for example, carrying and borrowing). On the other hand, student 2 may be using the right strategies, but she fails to reach the correct answers because of her impaired fact retrieval. In fact, multiplication facts retrieval (task 5 of the DeDiMa battery) is the task that best predicts the written calculation performance according to the model validation and battery reliability analyses conducted.

A more qualitative analysis of the students' observable processes involved in the response to mathematical tasks, through structured and clinical interviews, leads to confirmation or re-elaboration of the hypotheses advanced, in the cases of students we work with during remedial interventions.
The DeDiMa battery 


\section{CONCLUSIONS}

We have introduced the DeDiMa battery as an important tool of assessment, together with our validated 4-dimensional model, for evaluating mathematical learning difficulties. In particular, we have shown how an initial mathematical learning profile of a student can be outlined through these tools. The two profiles presented were of students with matching scores on a standardized test for diagnosing MLD, match-
Giannis Karagiannakis, Anna Baccaglini-Frank ing non-verbal IQ and mathematical experiences in school, so they would have generically been classified as "having MLD" and being quite similar. Instead, the assessment tool and model we presented allowed us to show how their mathematical learning profiles were actually quite different. In fact, it is likely that the same remedial intervention program for both of them would have led to very different (and non-optimal) outcomes in the two cases. We also remark on how these differences can become evident only through multi-dimensional tools like the ones proposed, accompanied by single score standardized measures.

The hypotheses we advanced based on this first administration of the DeDiMa battery helped to outline the students' profiles and better explain other aspects of their performances. Finally, we note that such hypotheses can later be confirmed or modified based on interactions with the students during remedial interventions and clinical interviews. One of our long-term aims is to explore the potential of this framework for adapting existing and designing new didactical material and activities, matching students' profiles, to establish more effective individualized teaching practices.

\section{ENDNOTES}

1 The battery was programmed in the $\mathrm{C}++$ language using the open-source cross-platform application framework QT version 4.7 and the open-source GNU compiler gcc. All the functions were implemented using generic QT/C++ approaches, so that the same code can be compiled for different operating systems (OS) such as Windows, Mac OS $X$ and Linux, with only minor differences in the appearance. The actual battery of tasks was executed on Windows machines.

2 When the battery is administered, the mediator can intervene to make sure the student is taking the test appropriately, and to encourage the student to respond as accurately and as quickly as s/he can. The software is designed to capture answers that are given randomly.

\section{REFERENCES}

Bartelet, D., Ansari, D., Vaessen, A., \& Blomert, L. (2014). Research in Developmental Disabilities
Cognitive subtypes of mathematics learning difficulties in primary education. Research in Developmental Disabilities, 35, 657-670.

Butterworth, B. (2010). Foundational numerical capacities and the origins of dyscalculia. Trends in Cognitive Sciences, 14, 534-541.

Fias, W., Menon, V., \& Szucs, D. (2013). Multiple components of developmental dyscalculia. Trends in Neuroscience and Education, 2, 43-47.

Fletcher, J. M., Lyon, G. R., Fuchs, L. S., \& Barnes, M. A. (2007). Learning disabilities: From identification to intervention. New York, NY: Guilford Press.

Geary, D. C., \& Hoard, M. K. (2001). Numerical and arithmetical deficits in learning-disabled children: Relation to dyscalculia and dyslexia. Aphasiology, 15, 635-647.

Gebuis, T., \& Reynvoet, B. (2011). Generating nonsymbolic number stimuli. Behavior Research Methods, 43, 981-986.

Grabner, R. H., \& Ansari, D. (2010). Promises and potential pitfalls of a 'cognitive neuroscience of mathematics learning'. ZDM Mathematics Education, 42, 655-660.

Heyd-Metzuyanim, E. (2012). The co-construction of learning difficulties in mathematics-teacher-student interactions and their role in the development of a disabled mathematical identity. Educational Studies in Mathematics, 83, 341-368. DOI: 10.1007/s10649-012-9457-z

Howard-Jones, P. A. (2011). A Multiperspective Approach to Neuro-educational Research. Educational Philosophy and Theory, 43, 24-30.

Karagiannakis, G., Baccaglini-Frank, A., \& Papadatos, Y. (2014). Mathematical learning difficulties subtypes classification. Frontiers in Human $\mathrm{Neu}$ roscience, 8,57 .

Karagiannakis, G., \& Cooreman, A. (in press). Focused intervention based on a classification MLD model. In: S. Chinn (ed.), The Routledge International Handbook of Dyscalculia and Mathematical Learning Difficulties - Routledge International Handbooks of Education.

Karagiannakis, G., Baccaglini-Frank, A., \& Roussos, P. (under review). Validation of a model for evaluating mathematical learning difficulties (working title).

Kaufmann, L. (2008). Dyscalculia: neuroscience and education. Educational Research, 50, 163-175.

Kaufmann, L., Mazzocco, M. M., Dowker, A., von Aster, M., Gobel, S. M., Grabner, R. H., Henik, A., Jordan, N. C., Karmiloff-Smith, A. D., Kucian, K., Rubinsten, O., Szucs, D., Shalev, R., \& Nuerk, H.-C. (2013). Dyscalculia from a developmental and differential perspective. Frontiers in Psychology, 4,516 .

Koumoula, A., Tsironi, V., Stamouli, V., Bardani, I., Siapati, S., Annika, G., Kafantaris, I., Charalambidou, I., Dellatolas, G., \& von Aster, M. (2004). An 
epidemiological study of number processing and mental calculation in Greek schoolchildren. Journal of Learning Disabilities, 37, 377-388.

Lanfranchi, S., Lucangeli, D., Jerman, O., \& Swanson, H. L. (2008). Math disabilities: Italian and US perspectives. Advances in Learning and Behavioral Disabilities, 21, 277-308.

Mazzocco, M. M. (2008). Defining and Differentiating Mathematical Learning Disabilities and Difficulties. In: D. B. Berch, \& M. M. Mazzocco (eds.), Why Is Math So Hard for Some Children? The Nature and Origins of Mathematical Learning Diffi-

The DeDiMa culties and Disabilities. Baltimore, MD: Brookes battery Publishing Company.

Panteliadou, S., \& Antoniou, F. (2008). Reading Test (in Greek). Greece: National Ministry of Education.

Siegler, R. S., \& Booth, J. L. (2004). Development of numerical estimation in young children. Child Development, 75, 428-444.

Siegler, R. S., \& Opfer, J. E. (2003). The development of numerical estimation: evidence for multiple representations of numerical quantity. Psychological Science, 14, 237-243.

Thorndike, R. L. (1982). Applied Psychometrics. Boston, MA: Houghton Mifflin.

Watson, S. M. R., \& Gable, R. A. (2013). Unraveling the Complex Nature of Mathematics Learning Disability: Implications for Research and Practice. Learning Disability Quarterly, 36, 178-187. 